\title{
Desarrollo local: reflexiones desde el desarrollo humano sostenible
}

\section{Cooperation and innovation in social economy companies}

\author{
Celso Vargas Elizondo' \\ Juan Rafael Hernández Navarro²
}

Fecha de recepción: 20 de enero del 2012

Fecha de aprobación: 19 de marzo del 2012

Vargas, C; Hernández, J. Desarrollo local: reflexiones desde el desarrollo humano sostenible. Tecnología

en Marcha. Vol. 25, № 6. Pág 93-103

I Licenciado en Filosofía, máster en Lingüística, doctor en Filosofía. Profesor e investigador de la Escuela de Ciencias Sociales del Tecnológico de Costa Rica. Profesor de la Universidad de Costa Rica y de la Maestría de Desarrollo Económico Local con énfasis en Turismo Comunitario (ITCR/FLACSO, C.R.) Teléfono: (506) 2550-228I. Correo electrónico: celvargas@itcrac

2 Licenciado en sociología por la Universidad de Costa Rica, con Maestría en Desarrollo Económico Local, FLACSO, CR-TEC. Profesor de la Escuela de Ciencias Sociales del Tecnológico de Costa Rica, actual coordinador de la Cátedra de Seminario de Estudios Costarricenses. Además, imparte cursos para la carrera de Gestión del Turismo Sostenible, del TEC. Teléfono: (506) 2550-20 I3. Correo electrónico: amora@itcr.ac.cr 


\section{Palabras clave}

Desarrollo humano, desarrollo humano sostenible, desarrollo económico local, desarrollo local, calidad de vida, indicadores, identidad, exclusión social.

\section{Resumen \\ El presente documento contiene una reflexión epistemológica y social del tema del desarrollo económico local, desde la perspectiva del desarrollo humano sostenible. En él se abordan problemas típi- cos del desarrollo; se establece su diferenciación con la expectativa de insinuar al menos las vías de acce- so a la condición de ciudadanía social, las amenazas que esta aspiración experimenta y un recorrido por diversas situaciones de inserción que se suelen presentar. Es, pues, un ensayo exploratorio con el objetivo de promover la reflexión teórica sobre este importante tema. Otorga un gran peso relativo a las estrategias orientadas a incluir económica, social y políticamente a los sectores más vulnerables, en actual proceso de globalización.}

\section{Key words}

Human development, sustainable human development, local economic development, local development, quality of life indicators, identity, social exclusion.

\begin{abstract}
This essay is a social and epistemological reflection on local economic development issued from the perspective of sustainable human development. It deals with some typical problems of development; and a differentiation is established expecting to suggest a road to the status of social citizenship and the threats that this aspiration conveys as well as an analysis of common insertion situations. As an exploratory essay, its main objective is to promote a theoretical reflexion on local economic development. At the same time, it gives an important relative weight to those strategies oriented to include that social sectors more vulnerable in the current process of globalisation.
\end{abstract}

\section{Introducción}

El concepto de desarrollo local plantea dos órdenes de problemas epistemológicos importantes. En primer lugar, se debe precisar claramente lo que se entiende por local y en segundo, es necesario esclarecer el significado de la palabra desarrollo. Aún más problemático resulta incluir el término desarrollo económico local.

Diversas posiciones reconceptualizan "local" $y$ "desarrollo" de diversas maneras. En este trabajo se analizan los conceptos de localidad y desarrollo a partir de la posición teórica del desarrollo humano sostenible. Se pretende explorar la aplicación de este marco general en la comprensión e intervención a nivel local. El trabajo se divide en tres secciones principales. En la primera se analiza el concepto de desarrollo. En la segunda, se da una aproximación al desarrollo por medio de indicadores tal y como aparecen, por ejemplo, en UN (2002). En la última sección se aborda el tema de lo local a partir del concepto de desarrollo discutido en la primera sección.

\section{Desarrollo humano sostenible}

Desarrollo humano

El desarrollo humano como tal, se separa de la visión de desarrollo de los 90, en que se utilizaba como sinónimo de "crecimiento económico". Ciertamente, el crecimiento económico y los indicadores asociados juegan un papel muy importante en términos de bienestar humano; pero es insuficiente para comprender el concepto desarrollo de manera integral.

Hay diferentes visiones sobre lo que debe ser el desarrollo centrado en el ser humano. Al decir "deber ser" se pretende indicar que el desarrollo humano es en esencia una perspectiva ética y estárelacionado con la realización histórica de un ideal de ser humano.

La adopción de este concepto por Naciones Unidas en 1990, basado en el trabajo de Amartya Sen (trabajo publicado independientemente en 1998) y

otros reconocidos economistas, se ha podido observar una evolución significativa del término, lo cual ha 
permitido vislumbrar algunas de las perspectivas desde las que puede ser abordardo.

Como ha señalado Griffith (2000), la introducción de este nuevo concepto de desarrollo surge como una toma de posición con respecto a la teoría dominante del desarrollo, basada en las relaciones de mercado; contexto en el cual el incremento de la producción de bienes y servicios, se supone, tiene una incidencia determinante en el mejoramiento de las condiciones económicas de los distintos países.

Igualmente implícito, es el supuesto de que el producto interno bruto (PIB) es una condición suficiente para determinar el grado de bienestar de la población. Además supone que la inyección de capitales en el sector privado con el marginamiento del Estado en su función distribuidora, generan un goteo o derrame desde la parte superior de la pirámide (la conocida teoría del derrame).

Históricamente, las bonanzas prometidas por los sustentadores de esa tesis, no se han cumplido, por el contrario, desde la década de 1980 nuestros países latinoamericanos han experimentado una creciente exclusión social y concentración de la propiedad y de la riqueza; fenómenos que se han intensificado en los últimos años, tal y como se pone de manifiesto en los Informes del Estado de la Nación y de la Región, para el caso de Costa Rica y de América Central y del Caribe.

Por otro lado, el dinamismo en términos económicos, de acceso a salud, empleo, infraestructura, educación, esparcimiento, entre otros, gravita alrededor de las metrópolis centroamericanas (ídem ant). En el caso de Costa Rica, las principales decisiones políticas son tomadas por la Casa Presidencial. Por lo tanto, la estrategia de desarrollo hacia afuera que apuesta a la atracción de capitales extranjeros, igualmente profundiza ese centralismo; consecuentemente, la descentralización política, económica y la promoción de estrategias de desarrollo local, una mayor autonomía en la gestión del territorio, son además de necesarias, imperativas.

Así pues, el concepto de bienestar fue entendido en términos exclusivamente económicos. Desde otros abordajes, el concepto de desarrollo humano va más allá del poder adquisitivo; así se define como "propiciar que la gente disponga de una gama mayor de opciones, que pueda hacer más cosas, vivir una vida más larga, eludir enfermedades evitables, tener acceso a la reserva mundial de conocimientos, etcétera" (Griffith, 2000, p.26).

Este primer modelo de desarrollo humano de 1990 pone énfasis en tres clases generales de derechos: I) el derecho a vivir una vida larga y saludable, 2) el derecho a una formación durante toda la vida y 3) el derecho a ingresos que le permitan tener una vida digna.

Así, según este modelo se puede observar que el logro de estos derechos está asociado al potenciamiento de un conjunto de capacidades de desarrollo individual. El Índice compuesto de Desarrollo Humano (IDH) pretende evaluar un conjunto de indicadores en cada una de las clases de derechos, de manera que el logro de ciertos mínimos permite asegurar que determinadas capacidades tienen una alta probabilidad de desarrollarse o no.

Entre los aspectos interesantes de este enfoque se encuentra la posibilidad de desarrollar otras aproximaciones y grupos de indicadores (como se verá en la tercera sección) divididos en tres clases, para medir otros ámbitos del desarrollo humano. Uno de los aspectos más importantes de dichos indicadores es haber permitido reconceptualizar la pobreza. Tradicionalmente, como señala Dubois (2000), el concepto "pobreza" estuvo relacionado con limitaciones en el ingreso. Pero en el marco del Desarrollo Humano se ha podido profundizar en la conceptualización de la pobreza, en términos de las privaciones o capacidades truncadas.

De esta manera, se elaboró un índice denominado "Indice de Privación de Capacidades" (IPC) de Naciones Unidas, el cual permite identificar algunas condiciones en las que dichas privaciones se pueden asegurar. Por ejemplo, en lo concerniente a la longevidad está el porcentaje de personas que morirán antes de los 40 años de edad; en cuanto al conocimiento, se maneja el porcentaje de personas analfabetas, y para la calidad de vida, se toma en cuenta el porcentaje de personas con acceso a los servicios de salud, agua potable, así como el porcentaje de desnutrición (Dubois, 2000).

\section{Desarrollo sostenible}

Este concepto de desarrollo humano puede ampliarse en varias vías de manera que los indicadores y principios de partida sean más aproximados a la situación real de los ciudadanos. Sin embargo, hay 
otra propuesta de desarrollo: el desarrollo sostenible. La formulación elaborada en estas páginas es compatible con la propuesta de la Agenda 21 de las Naciones Unidas.

De esta forma se podría entender el desarrollo sostenible como la evolución de una sociedad o de la sociedad global en el marco de un conjunto de restricciones, entendidas en el sentido matemático del término, es decir, como la delimitación del espacio o la evolución de la vida en cierta dirección. En torno al desarrollo humano, estas restricciones de sostenibilidad delimitan "el espacio" de posibilidades de las personas de continuar viviendo y de mejorar las condiciones materiales y espirituales. El potenciamiento de las capacidades humanas, la expansión de las libertadas individuales y de una convivencia armoniosa con el resto de las especies integrantes del planeta son el objetivo del desarrollo sostenible.

El potenciamiento de las capacidades humanas y de convivencia debe tomar en cuenta cuatro dimensiones: la económica considera todas aquellas variables relacionadas con las actividades de intercambio de bienes y servicios, así como las estructuras productivas de una colectividad. La social está relacionada con la vida de las personas en una colectividad y las formas en que pueden beneficiarse o no de su participación en esa colectividad, unidad territorial y a nivel global. Mientras que la dimensión institucional se relaciona con el conjunto de instituciones, regulaciones, mecanismos de participación, etc. que permiten potenciar o limitar la convivencia de las personas en el marco de esa colectividad o territorio. Finalmente, la dimensión ambiental se relaciona con el conjunto de recursos, estratos, etc. utilizables o que afectan a las personas o al planeta como sustentador de la vida.

\section{Desarrollo humano sostenible}

El desarrollo humano debe considerar estas cuatro dimensiones. En principio, ninguna de ellas tiene una mayor ponderación, aunque en situaciones específicas $\circ$ en determinados momentos alguna adquiere mucha mayor relevancia.

El desarrollo es un proceso dirigido y tiene como horizonte lo correcto y lo deseable. En este sentido, las restricciones deben imponerse al tipo de desarrollo deseado, deben moverse en una escala cuyos extremos estén formados, respectivamente, por lo que debe ser promovido o limitado; pues ambos, la promoción y la restricción cruzan las cuatro dimensiones. En cada dimensión hay aspectos o variables que deben ser promovidas por su importancia para el mejoramiento de la calidad de vida de las personas y de la vida en el planeta, pero también, y por las mismas razones, hay factores que deben ser limitados. Por ejemplo, son conocidas desde el Club de Roma, en la década de 1970, las necesidades de imponer restricciones a la tasa de consumo de recursos por las implicaciones que tiene, tanto para la economía misma como para el bienestar humano. De igual manera, determinadas modalidades de consumo vigentes en los países desarrollados deben ser variados por el enorme impacto que tienen sobre el futuro del planeta y de las nuevas generaciones.

Sin embargo, la producción de bienes y servicios ha mostrado ser fundamental para alcanzar niveles de independencia individual y colectiva, así como incrementar el bienestar de las personas. En este sentido, ambos aspectos, tanto la producción como el consumo, deben ser considerados a la hora de definir el horizonte humano al que hay que avanzar.

El desarrollo humano sostenible constituye un marco de reflexión con una gran riqueza conceptual. Como se ha señalado, se ha puesto énfasis en las condiciones externas necesarias para potenciar las capacidades humanas, las cuales están directamente relacionadas con condiciones las internas o subjetivas que, usualmente, miden la calidad de vida. Este concepto está relacionado con la identidad, con el "sentirse bien", con el arraigo y con el sentimiento de continuidad entre la valoración de sí mismo y el entorno.

A continuación se presenta una breve reflexión sobre algunos de los componentes de la calidad de vida.

\section{Calidad de vida}

Cuando la calidad de vida se analiza desde sus aspectos subjetivos, las emociones juegan un papel muy importante, pues elementos reconfiguradores que potencian o limitan las capacidades humanas. Se han reconocido tres clases generales de emociones: las de fondo, las emociones primarias y las secundarias [Damasio (1994)] Las emociones son expresión de condiciones internas a la vez de ser respuesta a condiciones externas. La manera de responder a las condiciones externas depende de la historia personal y de otros factores subjetivos 
como la motivación, la actitud ante la vida, el grado de satisfacción y el sentimiento de identidad.

Cuando se consideran las formas de vida que limitan el desarrollo de las capacidades humanas, es posible encontrar una gran diversidad, de ahí la necesidad de una tipificación de estas formas de limitación de la calidad de vida. Para los propósitos de este artículo se reconocerán dos: las reactivas y las carenciales. Ambas formas de vida no tienen por qu ser excluyentes, o presentarse de manera "pura".

Las formas de vida reactivas implican adaptación para responder a determinadas condiciones, lo cual en lugar de potenciar, limitará las posibilidades de realizarse como personas. Desde el punto de vista del desarrollo humano, es necesario esforzarse para superarlas.

Algunos ejemplos de este tipo de vidas reactivas son aquellas surgidas en el contexto de la inseguridad, es decir, sin ninguna seguridad de que puedan vivir el día siguiente; vidas en extrema contingencia y vulnerabilidad, pues dependen, literalmente, de otras personas. Estas situaciones son frecuentes en muchos países: vidas desarraigadas, personas desplazadas por motivos de guerra, persecución política o inestabilidad política y económica. Vidas que se desarrollan de manera infrahumana en los campos de refugiados.

El caso más dramático en los últimos años han sido los desplazados de Darfur, en Sudán, que no solamente deben protegerse de las etnias vecinas, sino que las mujeres tienen que dedicar una gran parte de su tiempo consiguiendo leña para la preparación de los alimentos, con un alto riesgo de ser violadas. Personas que sufren en silencio sin ninguna esperanza de encontrar una vida mejor. Personas que no han conocido otra forma de vida que esa durante muchos años.

Inmigrantes maltratados, discriminados en sus países de destino. Personas que viven en condiciones de pobreza sin ninguna esperanza de salir de ella. Nuestro globo está muy lejos de ser un lugar habitable para todos sus miembros. Estos grupos de personas viven una vida que no reúne las condiciones mínimas de calidad.

Otros casos de pobre calidad de vida en nuestros países, ubicables dentro de esta condición, son las personas excluidas por sexo (principalmente muje- res) por edad (niños y personas de la tercera edad), por condición socioeconómica y por origen étnico o capacidades disminuidas. Es difícil representarnos la tragedia humana que viven estos grupos.

Los tipos de vida carenciales expresan una carencia cultural, es decir, califica el tipo de identidad construíble en determinados contextos y bajo determinadas condiciones. Claramente, el tipo de identidad que se construya impone limitaciones o potencia el desarrollo individual o colectivo. Para poder caracterizar estas formas de existencia se debe introducir algunas consideraciones adicionales.

Según Gehlen (1987), la cultura es el conjunto de representaciones, organización de categorías y valores fundamentales para "completar" las carencias genéticas heredadas de la naturaleza. La construcción de cultura y de la identidad es un imperativo para todo ser humano. La cultura es el medio por el cual los individuos construyen su propia inserción en el mundo, en la sociedad, respondiendo a las inquietudes sobre temas de destino, sobre la responsabilidad del ser humano, entre otros. La identidad positiva, ese sentirse bien y aceptado, de contar con un lugar en sociedad posición desde la cual se satisfacen las necesidades espirituales, es una urgencia.

La cultura está ligada a la "esencia" misma del ser humano y a su individualidad, y de ahí que la tendencia natural de las culturas es hacia su diversificación, su pluralización. En las culturas se ponen de manifiesto las tendencias que Lersch (1962), denomina endotímicas: la necesidad de reconocimiento, el poder y el dejar constancia social e histórica de sus intereses. Estos tres factores endotímicos entran en conflicto y dinamizan la difusión de patrones culturales.

Sin embargo, la tipificación de las culturas por sus características dinámicas constituye solo un aspecto por considerar. Es necesario tomar en cuenta que las culturas tienden a permanecer en el tiempo. Hay una lucha perenne de las culturas por mantenerse, es decir, por tener continuadores. Las culturas tienden a exhibir componentes invariables que permiten comparar y ver diferencias con respecto a otras. Ahora bien, para que sea "exitosa" debe reunir una serie de condiciones como: flexibilidad, adaptabilidad, vitalidad, sentido y permanencia; no obstante, cuanta más permanencia tenga una cultura, menos dinámica es. 
Sin embargo, el mundo es más complejo que una sola cultura. En el proceso de globalización, aunque también dentro de lo estados nacionales, hay diversas culturas, de manera que una sociedad moderna es un tejido complejo de culturas con diferentes niveles y campos de acción. Tienen una estructura de capas, de superposiciones y de imbricaciones diversas.

Los colectivos deben construir su identidad o identidades en el marco de muchas alternativas, $y$ en las se enfrenta, como ha señalado la UNESCO (1996), una serie de tensiones, entre lo local y lo global, entre la tradición y la modernidad, entre el largo y el corto plazo, entre la multiplicación de conocimientos y su capacidad de asimilarlos.

Un factor que contribuye a incrementar las tensiones mencionadas son las tendencias globales las cuales fuertemente se contraponen a las tensiones culturales. De esta manera, es posible encontrar procesos de uniformización universal, como el control de calidad, la seguridad, la ciencia y la tecnología, entre otros, que tienden a oponerse a cualquier proceso de particularización. Estas tendencias globales participan en el proceso de proporcionar sentido a la vida de las personas, por lo cual, agudizan las tensiones mencionadas.

\section{Indicadores}

Uno de los principales éxitos de los modelos de desarrollo descrito radica en haber sido concebido para ser expresados en términos de indicadores. Un indicador tiene las siguientes propiedades: remite a una variable a la cual se le proporciona una base estadística que permite interpretar su correspondencia con el modelo y su aplicación. En tercer lugar, al ser una variable con metas fijables en rangos de mínimos y máximos, puede ser evaluada periódicamente. Existen otras propiedades importantes que estaremos presentando conforme avancemos en nuestra discusión. La finalidad de la elaboración y evaluación de los indicadores es proporcionar información precisa sobre los diferentes ámbitos de interés con el fin de agregarla en los distintos procesos de toma de decisiones.

Al inicio del artículo se presentaron dos modelos de desarrollo, los cuales no son incompatibles, sino que uno puede ser subsumido en el otro, tal como se propuso en la introducción del desarrollo humano sostenible. El primero de ellos, el modelo de desarrollo humano, utiliza tres temas principales, más uno agregado en los últimos años:

a) El disfrute de una vida longeva y saludable

b) El acceso al aprendizaje durante toda la vida

c) Contar con ingresos económicos que permitan llevar una vida digna

d) Ampliación de las oportunidades

Este modelo de análisis se aplica exitosamente cada año desde 1990, en los informes de Desarrollo Humano del PNUD. Cada uno de los temas se analiza en subtemas. A continuación un ejemplo con los temas a y $b$ :

Para el tema a)

i) Tendencias demográficas

ii) Recursos, acceso y servicios

iii) Agua, sanidad y nutricional estatus

iv) Inequidades en salud infantil y maternal

v) Crisis de salud global y riesgos

Para el tema b)

i) Compromisos con la educación: inversión pública

ii) Índice de escolaridad

iii) Tecnología: difusión y creación

(Fuente: HDR-2007-2008)

Una estructura similar se encuentra en el modelo de desarrollo sostenible, tal y como fue abordado por la Comisión de las Naciones Unidas (2002) para este propósito. Un total de 134 indicadores fueron propuestos y organizados en las cuatro dimensiones: la económica, la social, la institucional y la ambiental. Se trata de una visión integral de los principales aspectos asociados con las actividades humanas, como lo indica la Comisión de Desarrollo Sostenible:

"Esta integración conlleva el involucramiento de virtualmente todos los sectores tradicionales de la economía y de la actividad gubernamental, tales como el planeamiento económico, la agricultura, la salud, la energía, el agua, los recursos naturales, la industria, la educación y el ambiente, y así sucesivamente.... La asunción de integración es reflejada en los indicadores de desarrollo sostenible, los cuales contienen indicadores sociales, económicos, ambientales e 
institucionales, y deben ser tomados como mecanismos para la integración institucional, tales como los Consejos Nacionales de Desarrollo Sostenible, comités, misiones especiales, así como en las estrategias nacionales para el Desarrollo Sostenible. Esta aproximación fundamental al Desarrollo Sostenible debe ser considerada en el desarrollo, prueba y uso de los indicadores" (UN, 2002).

Una de las principales ventajas de este tipo de aproximación es su gran flexibilidad; pues según las prioridades nacionales, los indicadores a utilizar en cada uno de las dimensiones, temas y subtemas se pueden extender o reducir. Incluso se ha establecido una especie de estándar metodológico para la definición de nuevos indicadores. Así consta en los documentos de Naciones Unidas citados en la bibliografía.

Otra ventaja es que la metodología y estrategia propuestas pueden ser utilizadas en el desarrollo de nuevos conjuntos de temas e indicadores. Ya todos conocemos la fertilidad de este tipo de propuesta para la elaboración de indicadores alternativos como aquellos desarrollados en 1995 para medir la diferencias de ingreso según género, o los desarrollados en el contexto del año mundial sobre la prevención de desastres naturales. En este sentido, es una de las propuestas más exitosas, pues ha logrado plantear una alternativa a los modelos basados en el mercado.

No obstante, la ventaja principal observable en esta propuesta de desarrollo es que ha sido elaborada a partir de una visión ética, la cual parte del ser humano como centro del desarrollo. Asimismo, incorpora la dimensión de futuro, la evaluación de riesgos sociales, económicos, ambientales e institucionales y sus conexiones.

Finalmente, este enfoque ha permitido que a nivel mundial se puedan elaborar diferentes propuestas orientadas a lograr un mayor nivel de desarrollo. Además, se podría tomar en cuenta la limitación de riesgos asociados con los estilos de desarrollo actuales, la visualización de prácticas dentro del marco del desarrollo sostenible y un marco general que le daría cohesión. Adicionalmente, este enfoque incorpora la posibilidad de que el marco general evolucione adaptándose a las diversas situaciones que podemos encontrar.

Dicha evolución puede darse en dos sentidos diferentes. Primero, haciendo que los mínimos aceptables en cada uno de los temas, subtemas e indicadores sean cada vez más estrictos, además de ampliar los máximos. En segundo lugar, mediante la incorporación de nuevos temas e indicadores al núcleo básico de indicadores que permita adaptarse a las nuevas prioridades de los distintos países. Esto proporcionará información importante sobre aspectos de vital importancia para el desarrollo humano global.

El desarrollo humano sostenible proporciona, pues, un marco valorativo de gran importancia que debe ser tomado en consideración a la hora de definir estrategias de intervención que promuevan el potenciamiento de las capacidades humanas, tomando en consideración:

I. La medición del desarrollo

2. El concepto de desarrollo local

\section{Aproximación al concepto de desarrollo local}

El primer problema es definir la unidad espacial a la cual se le aplicará el concepto de "local", pues hay una diversidad de posiciones. En primer lugar, se pude partir desde un punto de vista metodológico: en el cuadrante positivo de un espacio cartesiano cuyo eje $x$ está formado por una escala que va de lo individual a lo global, mientras que el eje y está formado por una escala que va de una perspectiva sumativa de la sociedad, hasta una visión más holística de esta. Así, se puede expresar la visión sumativa en el sentido de que una colectividad es igual a la suma de los individuos que la forman.

En segundo lugar, otra posibilidad es a partir de la holística la cual defendería la posición de que el todo es mayor que la suma de sus partes. Los puntos de este espacio representarán las posiciones asumidas en relación con lo local y con la naturaleza de la colectividad. Es al menos lógicamente posible la existencia de un conjunto infinito de posiciones, sin embargo, la realidad dista mucho de esto pues hay posiciones limitadas. Así, la holística es una metodología descriptiva y clasificatoria que debe ser complementada con el análisis de las ventajas epistemológicas de cada una de las posiciones.

Ambas aproximaciones, con sus respectivos extremos pueden ser fácilmente confundidas. Llamaremos a este enfoque, posición monádica, en algún sentido similar al leibniziano. Todo lo demás es abstracción. Esta posición es claramente sumativa, no tiene 
mucho sentido hablar de una visión del individuo de manera holística.

En el otro extremo están las posiciones que mantienen todo lo interconectado mediante las llamadas leyes de la interconectividad universal. Lo real es este conjunto de relaciones establecidas a nivel universal; lo local entendido como cualquier fracción en esta totalidad es una abstracción, aun cuando se puedan reconocer zonas hegemónicas. Pero estas "fracciones" tienen sentido únicamente en el contexto de las relaciones universales establecidas, de las fuerzas de poder, de las contradicciones universales que se enfrentan, etc. Dichas posiciones son claramente holísticas, no es posible comprender un fraccionamiento de esta realidad por sí mismo, sin considerar las interconexiones con la totalidad; así esta posición será llamada universalista-holística.

El desarrollo humano sostenible, tal como ha sido caracterizado en las secciones anteriores, ubica su conceptualización de lo local a medio camino entre esas posiciones. Es un marco muy flexible que permite adaptarlo a diferentes unidades espaciales, pero no al individuo. En efecto, se requiere información de grupos de individuos con el fin de establecer comparaciones, pues no tendría mucho sentido aplicarlas al plano individual.

Esta es una propiedad heredada de las aproximaciones probabilísticas en las que el "caso singular" no tiene sentido, sino la manera en que un determinado "efecto" se distribuye en una colectividad, así la medición del desarrollo es, entonces, una especie de distribución de promedios en un grupo. En este sentido, la visión del desarrollo humano sostenible es un arreglo multivariable y multidimensional. Como se ha señalado ya, este enfoque se adapta a cualquier colectividad, desde una pequeña hasta una más global, permitiendo así la comparación.

En resumidas cuentas, el desarrollo humano sostenible se sitúa en una posición alejada de lo individual, admite un nivel importante de holismo al establecer criterios de clasificación de los individuos bajo determinadas métricas; pero a la vez, admite también un nivel adecuado de segregación. Es, pues, una posición que combina tanto el holismo como el sumativismo. Aplicado a la local, la comunidad puede ser un buen nivel para comenzar, pero es aplicable a otras formas de clasificación político-geográficas, como la división por provincia, cantones o distritos
(Atlas Cantonal, 2007)'. En este sentido, la definición de la unidad a la cual se le aplique el concepto de local es únicamente un problema de escala, excluyendo claramente al menos sus extremos.

Hay muchas situaciones que serían excluidas como ejemplos de desarrollo humano sostenible. Por ejemplo, los índices de desarrollo de una comunidad pueden mejorarse mediante la instalación de la empresa manufacturera internacional que proporcione empleo y acceso a nuevos servicios, pero esta situación aunque significativa, no garantiza sostenibilidad.

Para lograr sostenibilidad es necesario que la actividad se mantenga en el tiempo, además de cumplir con las condiciones ecológicas; lo anterior, por experiencia pasada, no ha sido así; tal es el caso de la pretendida explotación de oro a cielo abierto, de Industrias Infinito, en la zona norte de Costa Rica, conocido como crucitas. De esta manera, la mejor forma de mejorar el desarrollo local es por medio del potenciamiento de las capacidades de estas comunidades para generar nuevos emprendimientos, diversificar las actividades existentes y fortalecer la asociatividad con el fin de mejorar las condiciones sociales, institucionales y ambientales de la zona en cuestión.

En este sentido, el desarrollo humano sostenible debe ser complementado con una valoración de las alternativas que mejor garantizan la sostenibilidad de las capacidades generadas. Una forma abordarlo es mediante la diversificación. Por ejemplo, el mantenimiento de industrias de maquila podría tener un efecto importante sobre una comunidad, pero debe ser complementado dentro de un esquema más amplio de actividades económicas, sociales y culturales de las que participan los actores locales con el fin de enfrentar de la mejor manera las vulnerabilidades que puede enfrentar una comunidad; vale recordar las experiencias del muy conocido y denominado "capital golondrina" en la actividad maquilera en Centro América.

De igual manera, debe existir sincronización y armonía entre las políticas públicas nacionales, de los gobiernos locales y los grupos organizados, los cuales integrarían consideraciones sociales, económicas y ambientales.

I En Costa Rica, la República se divide administrativamente en provincias, cantones y distritos. 
Por otro lado, es necesario distinguir dos ámbitos relevantes. Por un lado, una de las medidas importantes del desarrollo está relacionado con la constatación de los servicios básicos de que dispone una comunidad, su calidad y su grado de acceso, pues así se pone de manifiesto el nivel de organización. Algunos ejemplos de los servicios son escuelas primarias, colegios de secundaria, programas de ayuda, servicios de salud, transporte público y empleo.

No puede dejarse de lado el hecho de que existe la generación de capacidades que intensifican las opciones disponibles, promueven la creatividad, innovación e identidad. No debe quedar por fuera la existencia de agencias de desarrollo local, ni el papel de las universidades con investigación, innovación, organización y cualificación de los emprendedores

\section{Concepto de desarrollo económico local}

El desarrollo local está directamente relacionado con el territorio, así como con el potenciamiento de las capacidades humanas de las personas que viven en una determinada región. El desarrollo local se representa mediante variables como las siguientes:

- Presencia de instituciones públicas eficientes

- Acceso a los servicios básicos (agua potable, alumbrado, transporte, telefonía...)

- Aspectos ambientales

- Oportunidades de empleo

- Seguridad

\section{El problema de las divisiones geográficas y políticas}

La noción más neutra quizá sea la de una comunidad. Sin embargo, en la medida en que se gana en especificidad se pierde la visión de las relaciones con otras unidades territoriales que dan como resultado una unidad mayor, es decir, se pierde generalidad.

Además, no es posible entender el desarrollo local sin mirar a condiciones extra-comunidad y extra país: hay una dependencia importante del comportamiento de la economía internacional y nacional necesaria sostener el desarrollo.

Una mejor manera de definir desarrollo local es como un conjunto de condiciones intra y extra comunitarias que inciden directamente en el logro de niveles de satisfacción de la población de un territorio en el que arraiga su identidad. Esas satisfacciones son de orden material, cultural, social, espiritual y donde la categoría ciudadano es tal vez la síntesis.

En el marco de esas tensiones indicadas, se pueden enumerar cuatro formas básicas de condiciones para la construcción de las identidades desde la perspectiva de considerar a los individuos en sus relaciones con colectividades y tendencias globales, sin dejar de lado que se pueden encontrar diversas combinaciones.

I) Identidad por uniformación. Este fenómeno se presenta cuando individuos o grupos construyen sus identidades subsumiendo los atributos 0 valores de los escalones superiores (o globales) de manera que hay un aporte individual muy reducido. La asimilación es el factor principal. En estos casos se puede hablar de alineación. Usualmente, estos individuos o grupos son muy sensibles a los cambios que ocurren en los niveles superiores por lo tanto, su identidad tiende a ser muy frágil y con una carga emotiva bipolar muy fuerte.

2) Identidad negativa. Los psicólogos, por ejemplo Krauskopft (2006), han descrito diferentes situaciones en las que los individuos, para construir sus identidades, adoptan como valores la negación (oposición), de aquellos valores dominantes sancionados por la sociedad. Estos grupos e individuos reaccionan contra el orden establecido y propugnan la edificación de un orden opuesto al existente. Este fenómeno lleva, en muchos casos, a un aislamiento de los grupos que en sociología se les conoce como contraculturales. No obstante, forman parte de la dialéctica de construcción de la identidad.

Ahora bien, desde la perspectiva de la ciencia política, bien podría interpretarse como una actitud de resistencia y reivindicación contra el imperio del mercado y de los grupos hegemónicos.

3) Exclusión. Desde la perspectiva de la identidad es muy difícil definir; pero puede establecerse de manera provisional como aquellas situaciones en la que individuos o grupos sienten extraños o inaprensibles los valores y principios adoptados por una unidad territorial o cultural. Así, el fenómeno de la exclusión puede darse de 
diferentes maneras: a nivel nacional, regional o global.

La exclusión se expresa como limitación al acceso a determinados conjuntos de beneficios sociales y culturales. Ahora bien, desde el punto de vista de la identidad, este fenómeno puede conllevar varios aspectos: la presencia simultánea de diversas perspectivas de identidad, en las que una es considerada como más valiosa, pero inaccesible por las condiciones en las cuales los individuos o grupos se encuentran.

Es frecuente encontrar este fenómeno en los grupos de inmigrantes al no poderse incorporar al nuevo país. En este sentido, no hay la posibilidad de lograr la integración de perspectivas de identidad en una sola o la consolidación de una de estas perspectivas. En muchos casos lleva de manera explícita o implícita a la negación de la cultura de origen. Sin embargo, el fenómeno de la exclusión va más allá; consiste en valorar como extraña, como inatinente una determinada cultura con la que se tiene cierta proximidad, o de la que se es sujeto excluido, como el caso de las maras, donde a falta de ciudadanía social y pertenencia, se satisface la afiliación matriculándose en las mismas. Desde una perspectiva socioeconómica, la exclusión social es una categoría de análisis; Pérez y Mora (2006) hablan de un bloqueo a la salida de la pobreza; de un núcleo duro e irreductible que a ciertos sectores en extrema exclusión, no les deja salida a esa condición.

4) Compatibilidad. Es aquella situación en la que un individuo o un grupo pueden integrar un conjunto de atributos de las escalas superiores (concepciones globales o regionales) con los atributos que le son propios del nivel donde esté. En este caso claramente, se resuelven las principales contradicciones presentables cuando se compara el nivel en el que se encuentra el individuo o el grupo con los niveles de orden superior.

Se observa compatibilidad cuando hay una serie de condiciones culturales o de otro tipo que posibilitan la construcción de una identidad sin renunciar a las particularidades del individuo o del grupo. El caso más interesante, desde el punto de vista normativo de la identidad, es en la que se alcance un alto grado de compatibilidad. Es este tipo de identidad el que se propone en este artículo.

En resumidas cuentas, hay tres formas de construcción de identidad carenciales en sentido importante. En la primera, se sacrifica lo individual para subsumirse en lo global. En la segunda, se protege al individuo negando cualquier aporte positivo de lo global o regional. En el tercero, más dramático, se excluye a determinados individuos de cualquier beneficio de estas tendencias globales. Claramente, en cada una de estas posibilidades hay grupos que ven truncadas sus posibilidades para desarrollar sus capacidades.

El contexto de las tensiones mencionadas anteriormente, debe ser afirmado en un marco de condiciones que permitan continuidad entre lo local y lo global. Este es uno de los grandes problemas por resolver.

\section{Conclusiones}

Para hablar de desarrollo humano sostenible es necesario pensarlo en términos de ciudadanía social, de acceso equitativo a esa ciudadanía, de pertenencia, de calidad de vida. La lógica de acumulación de nuestras sociedades, con especial referencia a Centro América, privilegia el desarrollo de las metrópolis (centralismo político, económico, de oportunidades), dejando relegadas a un segundo o tercer plano a las comunidades rurales y urbano marginales. La descentralización y la formulación de planes estratégicos en territorios definidos,constituye una estrategia alternativa o complementaria para el logro de una mejor calidad de vida.

La globalización usualmente percibida como avasalladora y homogenizadora, bajo ciertas condiciones deseables, puede brindar a los territorios un espacio de inserción exitosa en la globalización: lo "glocal" en palabras de Martin y Schuman (20I0)) posibilita una articulación de signo positivo entre lo local y lo global y, desde luego, con lo nacional, a partir de lo propio y lo diferente. Pero igualmente, la evidencia muestra que la existencia de "núcleos duros e irreductibles de la pobreza" (Pérez y Mora 2006) los cuales niegan la condición de ciudadanos sociales a un número importante de personas quienes merecen un compromiso y dedicación desde la política, desde la academia y desde todos los ciudadanos. 


\section{Bibliografía}

Dubois, A. (2000). La tensión entre medición y definición en el concepto alternativo de pobreza y bienestar del desarrollo humano. En En Ibarra y Unceta (2000).

Gehlen, A. (1987): El hombre. Salamanca. Ediciones Sígueme

Griffith, K. (2000). Desarrollo humano: Origen, evolución e impacto. En Ibarra y Unceta (2000).

Ibarra \& Unceta. (2000). Ensayos sobre el Desarrollo Humano. Barcelona, España: Icaria Editorial S. A.

Krauskoft, D. (2009) Adolescencia y Educación. San José, EUNED.

Lersch, P. (1962) La Estructura de la Personalidad. Editorial Scientia, España

Martin y Schumann (20I0) La Trampa de la Globalización. El ataque contra la Democracia y el Bienestar. Santillana S. A, Taurus. México

Naciones Unidas. (1993) Programa 21. Universidad Nacional de Costa Rica, Heredia

Pérez J. P. \& Mora M. (2006). De la pobreza a la exclusión social: La persistencia de la miseria en Centro América. Lugar: nombre de la editorial.Fundación Carolina.
PNUD/Costa Rica (2007) Atlas del desarrollo humano cantonal de Costa Rica, Universidad de Costa Rica. San Pedro.

Sen, A. (1992). Inequality Rexamined. New York: Oxford University Press.

Sen, A. (1999). Development as Freedom. Anchor Books, New York

UNESCO (1996) La Educación encierra un Tesoro (Informe Delors), Ginebra.

United Nations. (2002). Indicators of Sustainable Development: Guidelines and Methodologies. Documento disponible en Internet

United Nations Development Progrmme. (1990). Human Development Report 1990. Documento disponible en internet, sitio web del UNDP.

United Nations Development Progrmme. (1994). Human Development Report 1994. Documento disponible en internet, sitio web del UNDP.

Vargas, C. (2005). Ética y tecnología en el desarrollo humano. Libro Universitario Regional. Cartago, Costa Rica: Instituto Tecnológico de Costa Rica. 